\title{
Comparison of isotope pattern deconvolution and calibration curve quantification methods for the determination of estrone and $17 \beta$-estradiol in human serum
}

J. Pitarch-Motellón ${ }^{1}$, N. Fabregat-Cabello ${ }^{2,3}$, C. Le Goff ${ }^{2}$, A. F. Roig-Navarro ${ }^{1}$, J.V. Sancho-Llopis ${ }^{1}$, E. Cavalier ${ }^{2, *}$

${ }^{1}$ Research Institute for Pesticides and Water, Universitat Jaume I, Castelló, Spain

${ }^{2}$ Department of Clinical Chemistry, University of Liège, CHU Sart-Tilman, Liège, Belgium

${ }^{3}$ Current address: Hematology and Hemotherapy group, Health Research Institute Hospital La Fe (IIS La Fe), Valencia, Spain

*Corresponding author:

Pr. Étienne Cavalier, Service de Chimie Medicale, CHU de Liège, Domaine Universitaire du Sart-Tilman, 4000, Liege, Belgium, Phone: + 32 43667692, Fax: + 32 43667691,Email: Etienne.cavalier@chu.ulg.ac.be

Graphical abstract

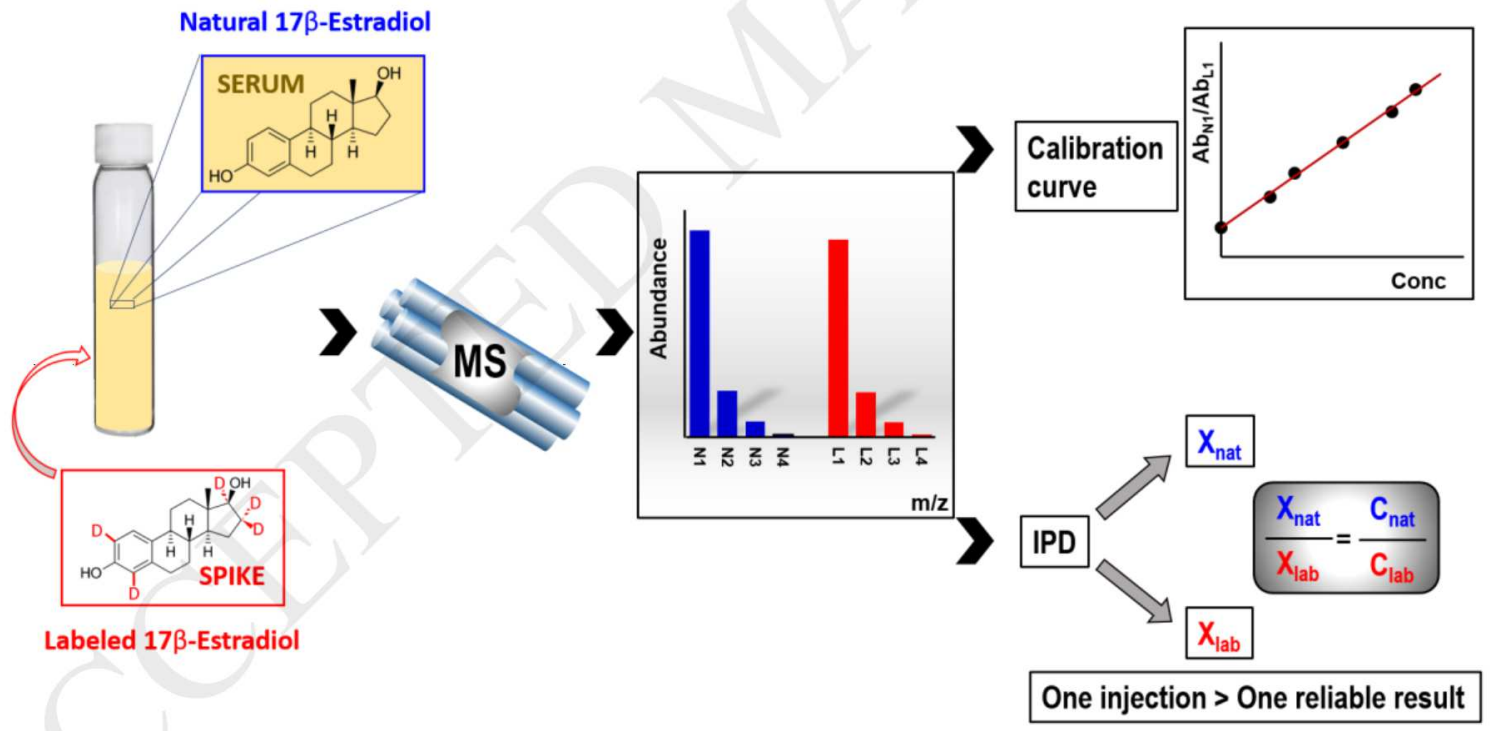

\section{Highlights}

- LC-MS quantification determination of estrone and $17 \beta$-estradiol in human serum 
- Classical calibration curve compared to multivariate isotope pattern deconvolution

- Methods successfully validated according to European medicines agency guidelines

- Both methods are suitable for routine analysis within the context of hospital's needs

- IPD has demonstrated to be faster and cost saving

\begin{abstract}
A Liquid Chromatography coupled to tandem mass spectrometry (LC-MS/MS) based method have been developed for the determination of the main estrogen compounds -estrone (E1) and 17ß-estradiol (E2)- in human serum. Two isotope dilution mass spectrometry (IDMS) quantification procedures have been used: a classical calibration curve-based method were compared to a recently developed isotope pattern deconvolution (IPD) method. IPD is based on isotopic abundance measurements and multiple linear regression. Validation was performed in terms of intra-assay repeatability $(n=5)$, inter-assay reproducibility $(n=9)$ and accuracy using spiked steroidfree serum at 5 concentration levels and 3 certified reference materials. Both methodologies meet EMEA requirements yielding recoveries between $79-106 \%$ and coefficient of variations of $1.7-8.3 \%$ along all experiments. Limits of quantification as low as $5 \mathrm{ng} / \mathrm{L}$ were achieved. 40 real samples were analysed for comparison purposes showing a great correlation between calibration and IPD concentration values. Real samples were also quantified by routine immunoassay analysis, which showed a significant proportional bias of 2.55 for E1 and good correlation for E2. While methods were considered suitable for routine or countercheck analysis within the context of hospital's needs, IPD has demonstrated to be faster and cost saving.
\end{abstract}

Keywords: estrone; $17 \beta$-estradiol; serum; LC-MS/MS; isotope dilution mass spectrometry; isotope pattern deconvolution 


\section{Introduction}

Estrogens are sexual steroidal hormones, derived from cholesterol, which are involved in a great number of gender-specific and non-gender-specific biological processes. The two main active estrogens in humans are estrone (E1) and $17 \beta$-estradiol (E2) and they are important in growth, nervous system maturation, bone structure and pregnancy, as well as playing a key role in breast cancer development [1-4].

Nowadays, there is an increasing interest in the simultaneous high-sensitivity and high throughput measurement of these estrogens for both clinical research and routine analysis. The main samples of interest include paediatric, pre-pubertal, postmenopausal and male serum samples. Unfortunately, most of immunoassays are not able to reach the very low levels needed in those cases. Only competitive radioimmunoassay (RIA) [5], which is associated with the health and safety risks of using radioactive materials, shows suitable limits of quantification. In general, immunoassay-based analysis often suffer from cross-reactivity with interfering endogenous components of the matrix, causing poor correlation with mass spectrometry-based methods, lacks of precision and accuracy, and only one estrogen is determined at a time. Thus, there is an increasing interest to shift towards stable isotope dilution (ID) in combination with Gas Chromatography and Liquid Chromatography coupled to mass spectrometry (GC-MS and LC-MS) or tandem mass spectrometry (MS/MS) methods for the determination of estrogens and other steroids [5-8].

Despite the known advantages of estrogen measurement by LC-MS/MS, which include increased sensitivity and specificity, and multiple determinations in one run their analysis is still challenging since these compounds do not contain highly ionisable functional groups. Thus, due to the extremely low concentration levels normally present in serum (often in the range of a few nanograms per liter), several approaches have been explored in the last years to tackle this problem.

Some work has been made in terms of extraction efficiency or chromatographic separation optimization. Two dimensional (2D) LC-MS/MS $[9,10]$ or automated exhaustive sample treatments $[11,12]$ that allow large sample volume injection while 
reducing background noise have been tested. Sensitivity is also enhanced sometimes by the addition of fluoride salts to the mobile phase. All those approaches need some complex instrumental equipment.

On the other side, derivatization of analytes is a widely-spread methodology in clinical analysis for the enhancement of sensibility by introducing a highly ionisable moiety in the structure. Recently, different derivatization agents have been tested [13-16]. Nevertheless, dansyl chloride is still the most used one because the derivative shows high ionisation efficiency, is obtained in aqueous solution with mild conditions, preventing unexpected hydrolysis processes, and can be directly injected in LC columns [17-21]. However, reduced selectivity is expected since charge remains in the dansyl moiety after collision-induced fragmentation and the measured MS transitions are not compound specific.

After derivatization, rigorously validated ID-LC-MS/MS methods provide reliable results for estrogen determination in serum. Classically, isotope dilution mass spectrometry (IDMS) is used along with calibration curve to avoid any matrix effect or instrumental drift, in a procedure that is time consuming. An alternative calculation in IDMS based on the measurement of isotopic abundances, isotope pattern deconvolution (IPD), can be used [22]. When the labelled internal standard (IS) is added to the sample, the altered isotopic abundances in the blend are a linear combination of those from the natural and from the labelled analogues. A deconvolution calculation using multiple linear regression provides the concentration of the natural compound in the sample. IDMS with IPD is a reliable, fast, and less expensive method. IPD provides one result per injection with accuracy, enhances the throughput of the whole method and it has already been successfully applied to different compounds and complex matrices. Namely, endocrine disrupting compound in wastewater $[23,24]$, or endogenous steroids in human urine [25-27].

In this work, we present the development and validation of a method for the determination of E1 and E2 in serum by LC-MS/MS according to the European Medicines Agency's (EMEA) guidelines [28]. Conventional calibration curve with internal standard quantification (the MS based method routinely applied in the laboratory) is thoroughly compared with the newly developed IPD calculations. With 
this new IPD quantification methodology, used for the first time to estrogen determination in serum, accurate, precise and less expensive analysis are expected.

\section{Materials and Methods}

\subsection{Materials}

Standard solutions of $17 \beta$-estradiol (E2) and $17 \alpha$-estradiol were purchased from LGC Standards (Luckenwalde, Germany). Standards solutions of estrone (E1), ${ }^{13} \mathrm{C}_{3}$-estrone $\left(E 1-{ }^{13} C_{3}\right)$ and ${ }^{2} \mathrm{H}_{5}-17 \beta$-estradiol $\left(E 2-D_{5}\right)$ were purchased from Cerilliant (Round Rock, TX, USA). All stock solutions were stored at $-20^{\circ} \mathrm{C}$ and employed to prepare gravimetrically diluted working standard solutions in acetonitrile. DC Mass Spect Gold MSG4000 ultra-low steroids and hormones human serum was purchased from Golden West Biological (Temecula, CA, USA). Certified reference materials BCR 576, BCR 577 and BCR 578 consisting in lyophilized serum with different E2 concentrations where obtained from the Institute for Reference Materials and Measurements (Geel, Belgium).

Dansyl chloride (Dns-Cl) and carbonate-bicarbonate buffer were purchased from Sigma-Aldrich (St. Louis, MO, USA). Formic acid for LC was from Fischer Chemicals (Loughborough, UK). All solvents used were LC-MS grade, water, acetonitrile, ethyl acetate and methyl-tert-butyl ether (MTBE) were from Biosolve BV (Valkenswaard, The Netherlands).

Standard stock solutions were prepared by gravimetrical dilution of certified $1 \mathrm{mg} / \mathrm{mL}$ solutions to approximately $50 \mu \mathrm{g} / \mathrm{mL}$, writing down the exact concentrations. Subsequent solutions were also prepared by gravimetrical dilution. Dansyl chloride 1 $\mathrm{mg} / \mathrm{mL}$ solution was prepared by dissolving exactly $25 \mathrm{mg}$ in $25 \mathrm{~mL}$ of acetonitrile while the carbonate/bicarbonate buffer $0.05 \mathrm{M}$ was prepared by dissolving one capsule in $100 \mathrm{~mL}$ of water LCMS and adjusting the $\mathrm{pH}$ to 10.5 by addition of a $\mathrm{NaOH}$ solution.

\subsection{LC-MS/MS Conditions}

Identification, characterization and quantification of the derivatized compounds was carried out with an UHPLC system using a Nexera x2 UPLC (Shimadzu, Kyoto, Japan) coupled with a quadrupole-linear ion trap QTRAP 6500 system (AB Sciex, Foster City, CA, USA) equipped with an IonDrive ${ }^{T M}$ Turbo $V$ interface. Chromatographic separation 
was tested with two different core-shell columns: Kinetex F5 100A $(1.7 \mu \mathrm{m}, 100$ x 2.1 $\mathrm{mm}$ ) and Kinetex C18 100A (2.6 $\mu \mathrm{m}, 100 \times 3 \mathrm{~mm})$. Separation was finally achieved with the C18 column fitted with a C18 AJ0-8775 guard column (all from Phenomenex, Torrance, CA, USA). The column oven was kept at $40^{\circ} \mathrm{C}$, injection volume was $30 \mu \mathrm{L}$ and samples were kept at 8 으 in the autosampler. Mobile phases consisted in $\mathrm{H}_{2} \mathrm{O}$ containing $0.01 \% \mathrm{HCOOH}$ as phase $\mathrm{A}$, and $\mathrm{ACN}$ containing $0.01 \% \mathrm{HCOOH}$ as phase $\mathrm{B}$. Gradient conditions were applied as follows: 50\% B (0-0.5 min), linear increase to 70\% $B$ in $0.1 \mathrm{~min}$, linear increase to $95 \%$ B in $4.4 \mathrm{~min}, 95 \%$ B (5-6 min), 50\% B (6.1-7.5 min). In order to protect the instrument from matrix contaminations, two cuts controlled by a divert valve were programmed at the beginning and at the end of the chromatographic run, allowing only the 2.5 to 6.5 min section to reach the mass spectrometer.

Analytes were ionized by electrospray in positive mode (ESI+) and detected by MS/MS in selected reaction monitoring (SRM). Optimum signal was obtained with the following parameters: $500^{\circ} \mathrm{C}$ as source temperature, curtain gas at $30 \mathrm{psi}$, collision assisted dissociation at $12 \mathrm{psi}$, IonSpray at $5.5 \mathrm{kV}$, nebulizer gas (GS1) at 30 psi and 60 psi of hot air through the Turbo $\mathrm{V}^{\mathrm{TM}}$ heaters (GS2). A dwell time of 25 ms was selected. Component-dependent parameters are shown in Table 1.

Analyst software version 1.6.2 (Sciex) was used for data acquisition. MultiQuant version 3.0.2 (Sciex) was used for data treatment and quantification.

\subsection{Sample preparation}

Micro-solid phase extraction ( $\mu$ SPE) and supported liquid extraction (SLE), both with 96-well plates platform, and Liquid-liquid extraction (LLE) methods were tested for estrogen extraction from serum at different concentrations. Ethyl acetate and methyl tert-butyl ether (MTBE) as eluent or acceptor phase were used. LLE was the extraction method definitely chosen. To $250 \mu \mathrm{L}$ of calibrators, controls or serum samples in $12 \times 75$ $\mathrm{mm} 5-\mathrm{mL}$ glass tubes, $10 \mu \mathrm{L}$ of labelled compound mix in acetonitrile were added, then samples were vortexed for $10 \mathrm{~s}$. Liquid-liquid extraction (LLE) was applied using $2 \mathrm{~mL}$ of MTBE, $10 \mathrm{~s}$ of vortex mixing and 15 min of mechanical agitation. In order to help in phase separation, samples were centrifuged at $3000 \mathrm{rpm}$ for $5 \mathrm{~min} .1 .7 \mathrm{~mL}$ of 
supernatant were transferred to clean 2-mL Eppendorf vials and evaporated under vacuum at 35 으.

Then, derivatization of analytes was carried out. To the dried extracts, $20 \mu \mathrm{L}$ of bicarbonate/carbonate buffer and $30 \mu \mathrm{L}$ of $1 \mathrm{mg} / \mathrm{mL}$ dansyl chloride in acetonitrile were added. Incubation was performed in a ThermoMixer (Eppendorf) at $60 \circ \mathrm{C}$ and $1000 \mathrm{rpm}$ for $10 \mathrm{~min}$. Derivatized extracts were diluted with $50 \mu \mathrm{L}$ of $\mathrm{H}_{2} \mathrm{O}$, transferred to LC vials with $0.2-\mathrm{mL}$ inserts and injected in the LC-MS system.

\subsection{Isotope Pattern Deconvolution}

Isotope pattern deconvolution (IPD) is an alternative approach of classical isotope dilution mass spectrometry methods, which are based on natural-to-labelled area ratios to build the calibration curve. In IPD, the natural distribution of abundances of the analyte of interest are altered by adding the corresponding isotope-labelled analogue and then multiple linear regression is used to mathematically deconvolute the resulting combined distribution of abundances to obtain the molar fractions [22]. Briefly, the abundance in the mix $\left(A_{m i x}^{S R M_{i}}\right)$, measured after the spike of labelled analogues to the natural occurring analytes, is a linear combination of the natural $\left(A_{n a t}^{S R M_{i}}\right)$ and labelled $\left(A_{\text {lab }}^{S R M_{i}}\right)$ compound abundances. In the case of $n$ measured transitions in the tandem mass spectrometry and using a single spike of labelled compound, this can be expressed in matrix form as follows:

$\left[\begin{array}{c}A_{\text {mix }}^{S R M_{1}} \\ A_{\text {mix }}^{S R M_{2}} \\ \vdots \\ A_{\text {mix }}^{S R M_{n}}\end{array}\right]=\left[\begin{array}{cc}A_{\text {nat }}^{S R M_{1}} & A_{\text {lab }}^{S R M_{1}} \\ A_{\text {nat }}^{S R M_{2}} & A_{\text {lab }}^{S R M_{2}} \\ \vdots & \vdots \\ A_{\text {nat }}^{S R M_{n}} & A_{\text {lab }}^{S R M_{n}}\end{array}\right]\left[\begin{array}{l}X_{\text {nat }} \\ X_{\text {lab }}\end{array}\right]+\left[\begin{array}{c}e^{S R M_{1}} \\ e^{S R M_{2}} \\ \vdots \\ e^{S R M_{n}}\end{array}\right]$ (Eq. 1)

Where an error vector $\left(e^{S R M_{i}}\right)$ has to be included in order to solve the equation system. Then, multiple linear regression is applied to minimize the error vector and to obtain the molar fractions ( $X_{n a t}$ and $X_{\text {lab }}$ respectively) that fit better the measured abundances in the mix. Calculations can be conducted easily and automatically by any spreadsheet software. Finally, as the amount of labelled compound $\left(N_{l a b}\right)$ added to the sample is known, the amount of natural analyte $\left(N_{\text {nat }}\right)$ is obtained:

$N_{\text {nat }}=N_{\text {lab }} \frac{X_{\text {nat }}}{X_{\text {lab }}}($ Eq. 2) 
As it can be seen, no methodological calibration is needed for the quantification and one injection produces one result. It requires, though, performing a full characterization of both compounds in terms of their isotopomer distribution of abundances and the certification by reverse isotope dilution of the labelled standard solutions.

\section{Results and Discussion}

\subsection{Method development}

Initial tests were performed to try if the quantification could be carried out without the need of derivatization. Individual $500 \mu \mathrm{g} / \mathrm{L}$ standard solutions in methanol/water $(1: 1, v / v)$ of each compound were infused in the ESI-MS/MS system in both positive ( $0.01 \%$ of formic acid was added) and negative modes. Instrumental conditions were optimized and the results indicated that sensitivity was insufficient for the desired lower limits of quantification. Thus, derivatization was deemed necessary. Compound-dependent instrumental conditions were optimized by means of the

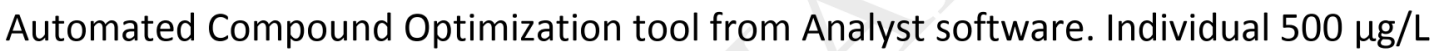
dansylated standard solutions in acetonitrile/water (30:70, v/v) were infused in the ESI-MS/MS system in positive mode at a rate of $7 \mu \mathrm{L} / \mathrm{min}$. Firstly, MS full-scans were obtained to select and optimize the precursor ion $\left([\mathrm{M}+\mathrm{H}]^{+}\right.$in all cases), followed by MS/MS scans to determine the transitions to acquire and to optimize the fragmentation parameters. Ionization source conditions were automatically optimized using Automated Source Optimization by injecting the same standards in Flow Injection Analysis (FIA) mode. The results obtained for each compound were compared and compromise values were selected in the cases where optimal values were different between analytes.

In order to achieve the chromatographic separation of all analytes and isobaric interferences, two chromatographic columns were tested under different gradient conditions and compositions, including methanol and acetonitrile with different combinations and concentrations of $\mathrm{NH}_{4} \mathrm{COOH}$ and $\mathrm{HCOOH}$. A Kinetex F5 pentafluorophenyl column (multiple interactions) was initially tested and optimized, which lead to a 9-min water/methanol gradient modified with $0.1 \% \mathrm{HCOOH}$ plus 0.2 $\mathrm{mM} \mathrm{NH}_{4} \mathrm{~F}$. Although the resulting chromatographic method was able to separate 
properly all analytes, including $17 \alpha$-estradiol (tested as isobaric interference of E2), the peaks presented bad chromatographic shape due to an excessive tailing in all analytes. Thus, the Kinetex C18 was tested, following the same optimization procedure, and the acetonitrile-based method described in the experimental section was found to be optimal. Although not being able to separate $17 \alpha$-estradiol from E2, as its occurrence in human serum has not been reported, the resulting 7.5-min gradient was used. For the extraction of analytes from the serum matrix, several approaches were tentatively assayed using standard solutions in water as well as spiked steroid-free serum at 5, 50 and $500 \mathrm{ng} / \mathrm{L}$ of E1 and E2. LLE, $\mu$ SPE and SLE methods using MTBE and ethyl acetate as acceptor phase or eluent were tested. For $\mu$ SPE and SLE methods, both as 96 -well plates, either resulted in bad recoveries or poor matrix elimination, with the increased difficulty of carrying out the subsequent derivatization step in the plates. Although being more time-consuming and laborious, LLE was chosen as it allowed near complete extraction ( $>90 \%$ recovery by absolute area when comparing the area in the extract versus the area of a standard of equivalent concentration) of the analytes and presented no problems in the following steps.

\subsection{Method validation}

The validation of the developed method was performed according to EMEA's "Guideline on bioanalytical method validation" [28].

For calibration-based quantification, calibration curves were prepared in steroid-free serum between 5 and $1000 \mathrm{ng} / \mathrm{L}$ of E1 and E2 (6 point plus blank -not spiked- and zero -spiked with IS only-samples), which showed deviations in the back-calculated concentrations below $15 \%$ respect the nominal value in at least 4 points in all cases (data not shown).

Validation standards were prepared in steroid-free serum at approximately 5, 10, 200, 400 and $800 \mathrm{ng} / \mathrm{L}$ of E1 and E2. In addition, additional validation assays were performed for E2 using three CRM: BCR 576, BCR 577 and BCR 578, with 31, 188 and $365 \mathrm{ng} / \mathrm{L}$ of E2 respectively (no certified concentrations of E1 were provided). Accuracy and precision were evaluated in terms of intra-assay repeatability $(n=5)$ and inter-assay reproducibility $(n=9)$ for all validation standards and CRM, by both calibration and IPD quantification approaches. All experiments with validation standards yielded 
recoveries between $79-118 \%$ and coefficient of variations of $1.2-14 \%$ for both methodologies (Tables 2 and 3). Regarding the CRM determination, both methods gave concentrations in the range of $81-101 \%$ respect the certified values and CV below $5 \%$ in all cases, except in the inter-assay experiment for BCR576 by IPD were it resulted in $17 \%$ CV (Table 4).

The method's LOQ was established as the minimum concentration value that was successfully validated, i.e., $5 \mathrm{ng} / \mathrm{L}$ for both analytes and quantification methods. That decision was made based on the impossibility to obtain true blank samples to which spike controlled amounts of analytes, since the theoretically steroid-free serum materials did contain some small quantities. The blank concentrations were estimated from the quantification of the zero point of the calibration curve -which is spiked only with internal standard- by IPD. MSG4000 commercial serum was found to contain on average $1.69 \mathrm{ng} / \mathrm{L}$ of $\mathrm{E} 1$ and $2.78 \mathrm{ng} / \mathrm{L}$ of $\mathrm{E} 2$. Concentrations found are clearly below our methods' LOQ, however chromatographic peaks corresponding to the analytes were clearly differentiable from background. (Figure S1, supplementary material). Thus, after calculating the mean value for all the calibration blank points used during the validation, concentrations of E1 and E2 in the commercial serum were estimated. Subsequent quantifications of validation standards and nominal values of calibration curves were adjusted accordingly.

The occurrence of possible matrix effects was checked for hemolyzed, renal, icteric and lipemic serum samples, as well as for two random normal serum samples. Samples were spiked at 3 times the lower limit of quantification (3xLLOQ) and near the upper limit of quantification (nearULOQ), and then subjected to the proposed sample treatment along with non-spiked samples of each type. Ratios between areas corresponding to natural and labelled compound were calculated, subtracting the nonspiked value (blank with internal standard), and then compared with a reference standard prepared in methanol and subjected to the same treatment. Acceptance ranges were set at $85-115 \%$ of the reference ratio for nearULOQ experiments and 80 120\% for 3xLLOQ. Results are shown in Table 5 for all the different matrices and spike levels tested. Renal serum samples produced the greatest deviations from the reference ratios and the method was deemed unfit for such samples. Hemolyzed serum sample spiked at 3xLLOQ also produced an abnormal recovery for E2, although 
the method performed well for E2 at a higher level and E1 at both levels. Similarly, performance in icteric samples was acceptable for E1 at high concentration and E2 at both levels, but unacceptable for E1 at low level. Finally, the method was suitable for E2 in lipemic samples but not for E1. Overall, no significant matrix effects were found in untreated samples, which were of main interest for the study.

\subsection{Method comparison}

Once the developed method was proved to meet the requirements of EMEA guidelines for both quantification procedures a thorough comparison was conducted. To this end, the methods were applied to the analysis of 40 real serum samples, including men and women of age comprised between 7 and 81 years old, randomly and anonymously selected. Samples were previously analysed by RIA (E1) or electrochemiluminiscence immunoassay (ECLIA) (E2) methods using the established procedures of the hospital's routine laboratories (LOQ around $17 \mathrm{ng} / \mathrm{L}$ for both approaches).

Passing-Bablok regression analysis was performed with open-source software, $\mathrm{cp}-\mathrm{R}$ [29]. The correlation between the newly developed LC-MS/MS method based on calibration curve versus the Hospital's reference RIA determination for E1 resulted in a proportional bias (slope) of 2.55 and constant bias (intercept) of $-9.41 \mathrm{ng} / \mathrm{L}$ (Figure 1A). Meanwhile, E2 LC-MS/MS calibration determinations suitably correlate against ECLIA, resulting in a proportional bias of 1.04 and constant bias of $1.44 \mathrm{ng} / \mathrm{L}$ (Figure 1B). The differences observed in the analysis of E1 by LC-MS respect the routine analysis performed by RIA are not really unexpected. They could be caused by interferences competing in the bonding phase of the immunoassay procedure. Therefore, the implementation of a new quantification method for routine analysis would require a complete characterization of the population's normal ranges of estrone in order to be able to properly assess future diagnosis and carry out steroidal monitoring tasks. On the other hand, the two MS-based methodologies were also compared. The proposed IPD method correlates properly with calibration-based quantification. Regression analyses presented $R^{2}$ values between 0.98 and 0.99 . However, IPD provided slightly lower concentration values with proportional biases of 0.83 and 0.73 for E1 and E2 respectively, while constant biases were -3.19 and $0.47 \mathrm{ng} / \mathrm{L}$ (Figure 2A 
and B). These results agree with the previously observed trends in recovery values obtained with validation standards and CRMs (Tables 2-4).

The seemingly better values provided by calibration curve quantification respect those of IPD may be caused by either the process of certification of standards and the presence of analytes in the theoretically steroid-free serum employed for this study. Existence of estrogens in the blank serum do not affect calibration curve procedure as calibration and validation standards are prepared in the same way. However, if not properly quantified and taken into account, the effect on measured abundances and IPD calculations can be significant. Thus, real free-steroid or accurately quantified ultra-low steroids matrix must be used. Regarding unsuitable certification of standards, the re-certification of labeled standards by IPD and reverse isotope dilution (RID) using suitable certified reference materials, has been proved as a correct way to improve accuracy [30]. Moreover, higher bias for E2 and IPD when compared with calibration can also be affected by deuterium scrambling problems. Losses of labile deuterium atoms during sample treatment or ionisation that can produce severe quantification problems in IPD isotopic abundance based calculations can occur for the labelled IS E2$D_{5}$.

Finally it should be emphasized that both quantification methods meets the EMEA guidelines but IPD entail substantial savings in time and money (quantity of standards used), since one injection provides one result.

\section{Conclusions}

In this work we present the development of two LC-MS quantification methods for the simultaneous determination of estrone (E1) and 17 $\beta$-estradiol (E2) in human serum, sharing the same sample treatment.

Validation with laboratory prepared standard solutions in free-steroid serum and with CRM BCR 576, 577 and 578 (human serum matrix of different concentrations) showed that both methods meet EMEA guidelines requirements in terms of precision, accuracy and matrix effect compensation. The methods were applied to real samples and compared between them and against the individual reference routine analysis based on immunoassay (RIA for E1 and ECLIA for E2) by Passing-Bablok regression analysis. The novel isotope dilution quantification method, namely isotope pattern 
deconvolution (IPD), provided very similar results to those of calibration-based method. However, some bias related with the presence of low concentrations of estrogens in the theoretical steroid-free serum and the low accurately certified standards is shown. This low bias is regarded as not significant since both methods meet validation requirements. LC-MS/MS calibration-based method presented good correlation against immunoassay for E2 but not for E1, were a proportional bias of 2.55 was found respect immunoassay analysis. These findings agree with the raising awareness of the need to re-evaluate the validity and suitability of bioassay methods for the analysis of estrogens due to their tendency to suffer from interferences and cross-reactivity. In addition, lower limits of quantification were achieved ( $5 \mathrm{ng} / \mathrm{L}$ versus $17 \mathrm{ng} / \mathrm{L}$ ) while needing a relatively small volume of sample of only $250 \mu \mathrm{L}$. Finally, IPD is shown as a rapid, less expensive, precise and exact method suitable for its application to estrogens in serum as a way to countercheck adverse or disagreeing results between conventional calibration-based and immunoassay methodologies, as well as being posed as a fully functional quantification method.

Acknowledgements: J. Pitarch-Motellón acknowledges the COST Action ES1307 for the STSM grant that made possible his stay at the CHU Sart-Tilman of Liège to carry out this study.

\section{Funding}

This research did not receive any specific grant from funding agencies in the public, commercial, or not-for-profit sectors. 


\section{References}

[1] T. Yasui, H. Uemura, M. Tezuka, M. Yamada, M. Irahara, M. Miura, T. Aono, Biological Effects of Hormone Replacement Therapy in Relation to Serum Estradiol Levels, Horm. Res. Paediatr. 56 (2002) 38-44. doi:10.1159/000048088.

[2] B. Ettinger, A. Pressman, P. Sklarin, D.C. Bauer, J.A. Cauley, S.R. Cummings, Associations between Low Levels of Serum Estradiol, Bone Density, and Fractures among Elderly Women: The Study of Osteoporotic Fractures 1, J. Clin. Endocrinol. Metab. 83 (1998) 2239-2243. doi:10.1210/jcem.83.7.4708.

[3] M. Hill, A. Pašková, R. Kančeva, M. Velíková, J. Kubátová, L. Kancheva, K. Adamcová, M. Mikešová, Z. Žižka, M. Koucký, H. Šarapatková, V. Kačer, P. Matucha, M. Meloun, A. Pařizek, Steroid profiling in pregnancy: A focus on the human fetus, J. Steroid Biochem. Mol. Biol. 139 (2014) 201-222. doi:10.1016/j.jsbmb.2013.03.008.

[4] F.Z. Stanczyk, B.W. Mathews, M.E. Sherman, Relationships of sex steroid hormone levels in benign and cancerous breast tissue and blood: A critical appraisal of current science, Steroids. 99 (2015) 91-102. doi:10.1016/j.steroids.2014.12.011.

[5] I.A. Blair, Analysis of estrogens in serum and plasma from postmenopausal women: Past present, and future, Steroids. 75 (2010) 297-306. doi:10.1016/j.steroids.2010.01.012.

[6] L.M. McShane, J.F. Dorgan, S. Greenhut, J.J. Damato, Reliability and validity of serum sex hormone measurements., Cancer Epidemiol. Biomarkers Prev. 5 (1996) 923-8. http://www.ncbi.nlm.nih.gov/pubmed/8922302.

[7] A.W. Hsing, F.Z. Stanczyk, A. Belanger, P. Schroeder, L. Chang, R.T. Falk, T.R. Fears, Reproducibility of Serum Sex Steroid Assays in Men by RIA and Mass Spectrometry, Cancer Epidemiol. Biomarkers Prev. 16 (2007) 1004-1008. doi:10.1158/1055-9965.EPI-06-0792.

[8] A.S.P. Boggs, J.A. Bowden, T.M. Galligan, L.J. Guillette, J.R. Kucklick, Development of a multi-class steroid hormone screening method using Liquid Chromatography/Tandem Mass Spectrometry (LC-MS/MS), Anal. Bioanal. Chem. 408 (2016) 4179-4190. doi:10.1007/s00216016-9512-1.

[9] T. Fiers, B. Casetta, B. Bernaert, E. Vandersypt, M. Debock, J.-M. Kaufman, Development of a highly sensitive method for the quantification of estrone and estradiol in serum by liquid chromatography tandem mass spectrometry without derivatization, J. Chromatogr. B. 893-894 (2012) 57-62. doi:10.1016/j.jchromb.2012.02.034.

[10] S. Pauwels, L. Antonio, I. Jans, A. Lintermans, P. Neven, F. Claessens, B. Decallonne, J. Billen, D. Vanderschueren, P. Vermeersch, Sensitive routine liquid chromatography-tandem mass spectrometry method for serum estradiol and estrone without derivatization, Anal. Bioanal. Chem. 405 (2013) 8569-8577. doi:10.1007/s00216-013-7259-5.

[11] L.J. Owen, F.C. Wu, B.G. Keevil, A rapid direct assay for the routine measurement of oestradiol and oestrone by liquid chromatography tandem mass spectrometry, Ann. Clin. Biochem. 51 (2014) 360-367. doi:10.1177/0004563213501478. 
[12] K.M. Wooding, J.A. Hankin, C.A. Johnson, J.D. Chosich, S.W. Baek, A.P. Bradford, R.C. Murphy, N. Santoro, Measurement of estradiol, estrone, and testosterone in postmenopausal human serum by isotope dilution liquid chromatography tandem mass spectrometry without derivatization, Steroids. 96 (2015) 89-94. doi:10.1016/j.steroids.2015.01.007.

[13] A. Khedr, A.M. Alahdal, Liquid chromatography-tandem mass spectrometric analysis of ten estrogen metabolites at sub-picogram levels in breast cancer women, J. Chromatogr. B Anal. Technol. Biomed. Life Sci. 1031 (2016) 181-188. doi:10.1016/j.jchromb.2016.07.051.

[14] $\mathrm{X} \mathrm{Li,} \mathrm{A.A.} \mathrm{Franke,} \mathrm{Improved} \mathrm{profiling} \mathrm{of} \mathrm{estrogen} \mathrm{metabolites} \mathrm{by} \mathrm{orbitrap} \mathrm{LC/MS,}$ Steroids. 99 (2015) 84-90. doi:10.1016/j.steroids.2014.12.005.

[15] P. Keski-Rahkonen, R. Desai, M. Jimenez, D.T. Harwood, D.J. Handelsman, Measurement of Estradiol in Human Serum by LC-MS/MS Using a Novel Estrogen-Specific Derivatization Reagent, Anal. Chem. 87 (2015) 7180-7186. doi:10.1021/acs.analchem.5b01042.

[16] W.C. Yang, F.E. Regnier, D. Sliva, J. Adamec, Stable isotope-coded quaternization for comparative quantification of estrogen metabolites by high-performance liquid chromatography-electrospray ionization mass spectrometry, J. Chromatogr. B Anal. Technol. Biomed. Life Sci. 870 (2008) 233-240. doi:10.1016/j.jchromb.2008.06.026.

[17] Y. Ke, J. Bertin, R. Gonthier, J.N. Simard, F. Labrie, A sensitive, simple and robust LCMS/MS method for the simultaneous quantification of seven androgen- and estrogen-related steroids in postmenopausal serum, J. Steroid Biochem. Mol. Biol. 144 (2014) 523-534. doi:10.1016/j.jsbmb.2014.08.015.

[18] X Xu, J.M. Roman, H.J. Issaq, L.K. Keefer, T.D. Veenstra, R.G. Ziegler, Quantitative measurement of endogenous estrogens and estrogen metabolites in human serum by liquid chromatography-tandem mass spectrometry, Anal. Chem. 79 (2007) 7813-7821. doi:10.1021/ac070494j.

[19] M.M. Kushnir, A.L. Rockwood, J. Bergquist, M. Varshavsky, W.L. Roberts, B. Yue, A.M. Bunker, A.W. Meikle, High-Sensitivity Tandem Mass Spectrometry Assay for Serum Estrone and Estradiol, Am. J. Clin. Pathol. 129 (2008) 530-539. doi:10.1309/LC03BHQ5̄XIPJEKG.

[20] S.S.-C.T. and, M.J. Welch, Development and Evaluation of a Reference Measurement Procedure for the Determination of Estradiol-17 $\beta$ in Human Serum Using Isotope-Dilution Liquid Chromatography-Tandem Mass Spectrometry, (2005). doi:10.1021/AC050837I.

[21] R.E. Nelson, Liquid Chromatography-Tandem Mass Spectrometry Assay for Simultaneous Measurement of Estradiol and Estrone in Human Plasma, Clin. Chem. 50 (2004) 373-384. doi:10.1373/clinchem.2003.025478.

[22] J.I. García-Alonso, P. Rodríguez-González, IDMS procedures and calculation methods, in: Isot. Dilution Mass Spectrom., 2013: pp. 41-76.

[23] N. Fabregat-Cabello, J. Pitarch-Motellón, J. V. Sancho, M. Ibáñez, A.F. Roig-Navarro, Method development and validation for the determination of selected endocrine disrupting 
compounds by liquid chromatography mass spectrometry and isotope pattern deconvolution in water samples. Comparison of two extraction techniques, Anal. Methods. 8 (2016) 28952903. doi:10.1039/C6AY00221H.

[24] N. Fabregat-Cabello, J. V Sancho, A. Vidal, F. V González, A.F. Roig-Navarro, Development and validation of a liquid chromatography isotope dilution mass spectrometry method for the reliable quantification of alkylphenols in environmental water samples by isotope pattern deconvolution., J. Chromatogr. A. 1328 (2014) 43-51. doi:10.1016/j.chroma.2013.12.077.

[25] J. Pitarch-MotellÓn, A.F. Roig-Navarro, J.V. Sancho, M. Ibáñ̃z, N. Fabregat-Cabello, O.J. Pozo, R. Ventura, J.I. Garcĺa Alonso, P. Rodríguez-Gonzáez, A. González Gago, A. Ereño Artabe, P. Van Eenoo, K. Deventer, Y. Dehnes, S. Rzeppa, Evaluation of uncertainty sources in the determination of testosterone in urine by calibration-based and isotope dilution quantification using ultra high performance liquid chromatography tandem mass spectrometry, J. Chromatogr. A. 1508 (2017) 73-80. doi:10.1016/j.chroma.2017.05.072.

[26] J. Pitarch-MotellÓn, J.V. Sancho, M. Ibáñez, O. Pozo, A.F. Roig-Navarro, Determination of selected endogenous anabolic androgenic steroids and ratios in urine by ultra high performance liquid chromatography tandem mass spectrometry and isotope pattern deconvolution, J. Chromatogr. A. 1515 (2017) 172-178. doi:10.1016/j.chroma.2017.08.006.

[27] A. Ereño Artabe, A. González-Gago, A. Suarez Fernández, J. Pitarch Motellón, A.F. RoigNavarro, O.J. Pozo, P. Rodríguez-González, J.I. Garcĺa Alonso, Isotope dilution LC-ESI-MS/MS and low resolution selected reaction monitoring as a tool for the accurate quantification of urinary testosterone, J. Pharm. Biomed. Anal. 163 (2019) 113-121. doi:10.1016/j.jpba.2018.09.038.

[28] European Medicines Agency, Guideline on bioanalytical method validation, EMEA, Comm. Med. Prod. Hum. Use. 44 (2012) 1-23. doi:EMEA/CHMP/EWP/192217/2009.

[29] D.T. Holmes, cp-R, an interface the R programming language for clinical laboratory method comparisons, Clin. Biochem. 48 (2015) 192-195.

doi:10.1016/j.clinbiochem.2014.10.015.

[30] Pitarch-MotellÓn J, Fabregat-Cabello N, Le Goff C, Roig-Navarro AF, Cavalier E. Recertification of hydroxyvitamin $\mathrm{D}$ standards by isotope pattern deconvolution, J Chromatogr B. (2018). 
Figure captions

Figure 1.- Passing-Bablok regression analysis. Correlation between LC-MS/MS method based on calibration curve and RIA determination of E1 (1A) and ECLIA determinations of E2 (1B).
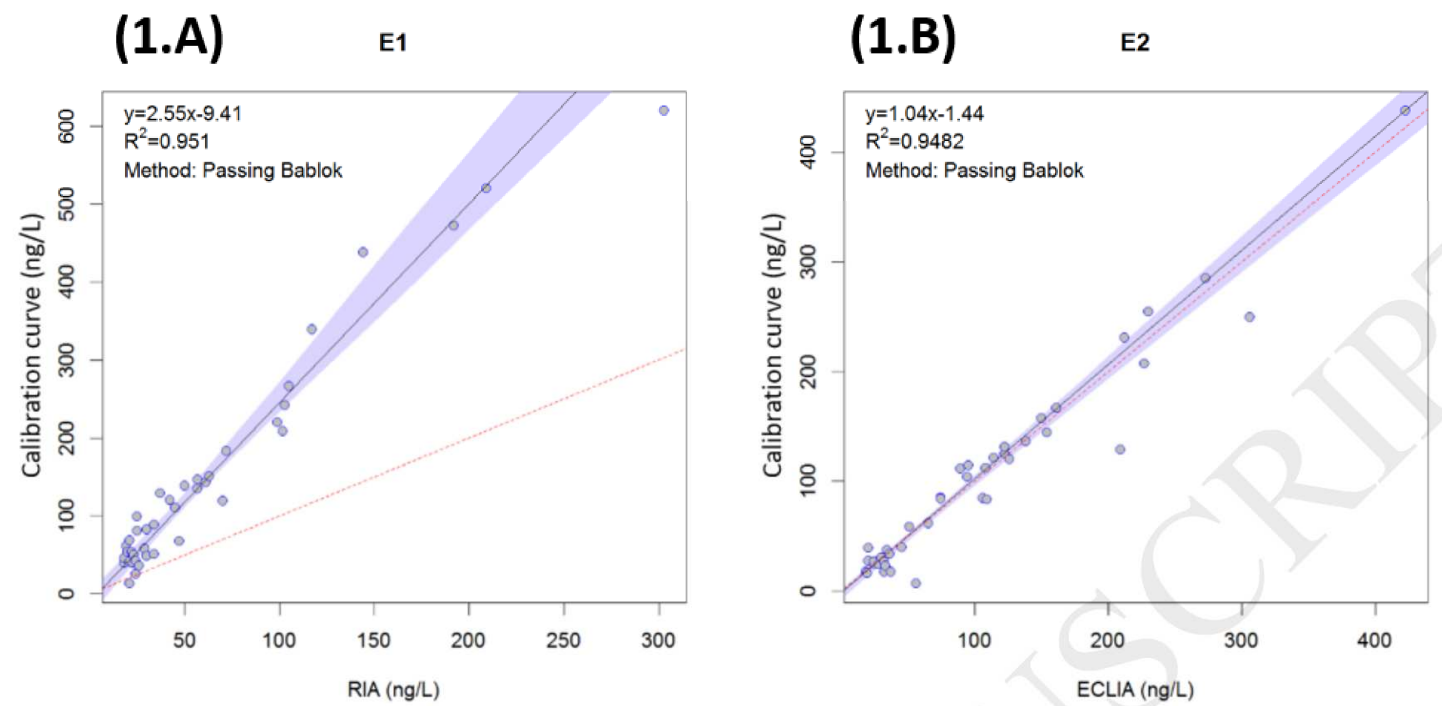

Figure 2.- Passing-Bablok regression analysis. Correlation between IPD and LC-MS/MS method based on calibration curve determination of E1 (2A) and E2 (2B).
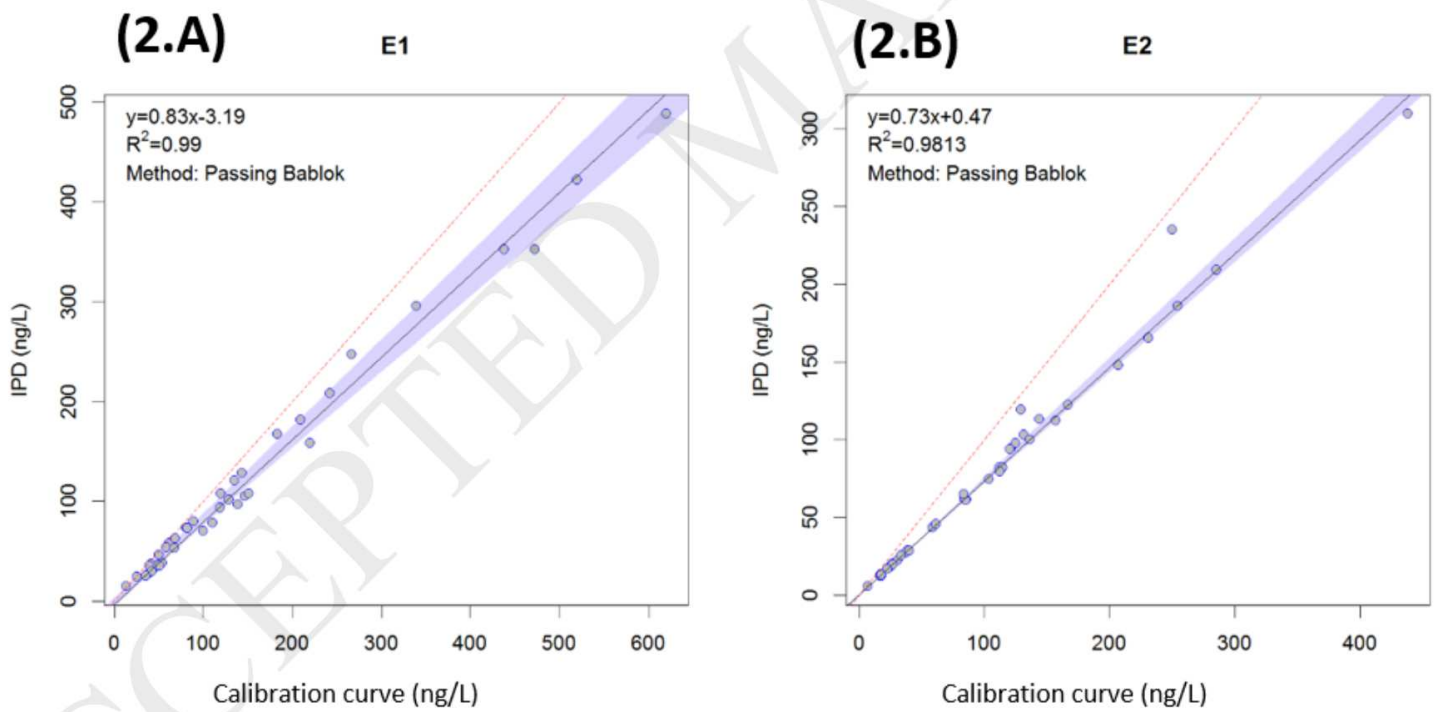
Table 1. Component-dependent MS parameters.

\begin{tabular}{|c|c|c|c|c|c|}
\hline Compound & $\mathrm{DP}^{1}$ & $E P^{2}$ & $C E^{3}$ & $\mathrm{CXP}^{4}$ & Transitions \\
\hline \multirow{3}{*}{ E1 } & \multirow{3}{*}{166} & \multirow{3}{*}{10} & \multirow{3}{*}{43} & \multirow{3}{*}{10} & $504>171$ \\
\hline & & & & & $505>171$ \\
\hline & & & & & $504>115$ \\
\hline \multirow{3}{*}{ E2 } & \multirow{3}{*}{151} & \multirow{3}{*}{10} & \multirow{3}{*}{43} & \multirow{3}{*}{12} & $506>171$ \\
\hline & & & & & $507>171$ \\
\hline & & & & & $506>115$ \\
\hline \multirow{2}{*}{ E1-13C3 } & \multirow{2}{*}{166} & \multirow{2}{*}{10} & \multirow{2}{*}{43} & \multirow{2}{*}{10} & $507>171$ \\
\hline & & & & & $508>171$ \\
\hline \multirow{2}{*}{ E2-D5 } & \multirow{2}{*}{151} & \multirow{2}{*}{10} & \multirow{2}{*}{43} & \multirow{2}{*}{12} & $511>171$ \\
\hline & & & & & $512>171$ \\
\hline \multicolumn{6}{|c|}{${ }^{1}$ Declustering Potential (V) } \\
\hline \multicolumn{6}{|c|}{${ }^{2}$ Entrance Potential (V) } \\
\hline \multicolumn{6}{|c|}{${ }^{3}$ Collision Energy (V) } \\
\hline${ }^{4}$ Collision Cell & Pot & (V) & & & \\
\hline
\end{tabular}

Table 2. Validation results for E1 in intra- and inter-assay experiments.

\begin{tabular}{ccccccccc}
\hline \multirow{2}{*}{ E1 } & \multicolumn{3}{c}{ Intra-assay (n=5) } & \multicolumn{3}{c}{ Inter-assay (n=9) } \\
\cline { 2 - 10 } Conc. (ng/L) & $\begin{array}{c}\text { Recovery } \\
(\%)\end{array}$ & CV & $\begin{array}{c}\text { Recovery } \\
(\%)\end{array}$ & CV & $\begin{array}{c}\text { Recovery } \\
(\%)\end{array}$ & CV & $\begin{array}{c}\text { Recovery } \\
(\%)\end{array}$ & CV \\
\hline 5 & 92 & $13.9 \%$ & 118 & $9.5 \%$ & 99 & $6.3 \%$ & 102 & $4.7 \%$ \\
\hline 10 & 96 & $6.5 \%$ & 116 & $6.0 \%$ & 102 & $5.4 \%$ & 113 & $5.9 \%$ \\
\hline 200 & 113 & $1.2 \%$ & 103 & $1.3 \%$ & 112 & $1.4 \%$ & 103 & $1.7 \%$ \\
\hline 400 & 106 & $2.0 \%$ & 100 & $2.1 \%$ & 107 & $4.6 \%$ & 102 & $4.9 \%$ \\
\hline 800 & 97 & $6.3 \%$ & 98 & $7.0 \%$ & 96 & $3.8 \%$ & 98 & $4.3 \%$ \\
\hline
\end{tabular}

Table 3. Validation results for E2 in intra- and inter-assay experiments.

\begin{tabular}{ccccccccc}
\hline \multirow{2}{*}{ E2 } & \multicolumn{3}{c}{ Intra-assay $(\mathrm{n}=5)$} & \multicolumn{3}{c}{ Inter-assay (n=9) } \\
\cline { 2 - 10 } Conc. (ng/L) & $\begin{array}{c}\text { Cal } \\
\text { Recovery } \\
(\%)\end{array}$ & CV & $\begin{array}{c}\text { Recovery } \\
(\%)\end{array}$ & CV & $\begin{array}{c}\text { Recovery } \\
(\%)\end{array}$ & CV & $\begin{array}{c}\text { Recovery } \\
(\%)\end{array}$ & CV \\
\hline 5 & 103 & $8.3 \%$ & 98 & $5.0 \%$ & 106 & $5.3 \%$ & 84 & $5.2 \%$ \\
\hline 10 & 102 & $4.4 \%$ & 95 & $5.3 \%$ & 105 & $2.5 \%$ & 88 & $2.7 \%$ \\
\hline 200 & 90 & $4.6 \%$ & 80 & $4.6 \%$ & 104 & $2.6 \%$ & 94 & $2.6 \%$ \\
\hline 400 & 92 & $3.1 \%$ & 79 & $2.9 \%$ & 105 & $3.3 \%$ & 94 & $3.4 \%$ \\
\hline 800 & 99 & $2.2 \%$ & 86 & $1.7 \%$ & 108 & $2.1 \%$ & 96 & $1.9 \%$ \\
\hline
\end{tabular}


Table 4. Validation results for E2 determination experiments in three certified reference materials.

\begin{tabular}{cccccccccc}
\hline \multirow{2}{*}{ CRM } & \multicolumn{4}{c}{ Intra-assay (n=5) } & \multicolumn{3}{c}{ Inter-assay (n=9) } \\
\cline { 2 - 12 } Code & $\begin{array}{c}\text { Conc. E2 } \\
\text { (ng/L) }\end{array}$ & $\begin{array}{c}\text { Recovery } \\
(\%)\end{array}$ & CV & $\begin{array}{c}\text { Recovery } \\
\text { (\%) }\end{array}$ & CV & $\begin{array}{c}\text { Recovery } \\
(\%)\end{array}$ & CV & $\begin{array}{c}\text { Recovery } \\
(\%)\end{array}$ & CV \\
\hline BCR576 & 31.05 & 97 & $5.2 \%$ & 85 & $5.4 \%$ & 101 & $3.6 \%$ & 84 & $17.0 \%$ \\
\hline BCR577 & 187.94 & 87 & $4.0 \%$ & 81 & $4.2 \%$ & 93 & $1.6 \%$ & 83 & $1.6 \%$ \\
\hline BCR578 & 364.99 & 94 & $3.8 \%$ & 88 & $4.0 \%$ & 101 & $3.7 \%$ & 91 & $4.2 \%$ \\
\hline
\end{tabular}

Table 5. Matrix effect in different types of serum, obtained by comparison with standards in solvent at the same concentration levels.

\begin{tabular}{|c|c|c|c|c|}
\hline \multirow[b]{2}{*}{ Sample type } & \multicolumn{4}{|c|}{ Matrix Effect (\%) } \\
\hline & $\begin{array}{c}\text { E1 at } \\
3 \times L L O Q\end{array}$ & $\begin{array}{c}\text { E1 at } \\
\text { nearUloQ }\end{array}$ & $\begin{array}{c}\text { E2 at } \\
3 \times L L O Q\end{array}$ & $\begin{array}{c}\text { E2 at } \\
\text { nearUloQ }\end{array}$ \\
\hline Serum A & 88 & 88 & 80 & 91 \\
\hline Serum B & 95 & 103 & 98 & 104 \\
\hline Hemolized & 99 & 94 & 171 & 106 \\
\hline Renal & 354 & 94 & 118 & 77 \\
\hline Icteric & 170 & 99 & 97 & 99 \\
\hline Lipemic & 121 & 81 & 97 & 92 \\
\hline
\end{tabular}

